\title{
THE NEUMANN PROBLEM \\ FOR THE 2-D HELMHOLTZ EQUATION IN A DOMAIN, BOUNDED BY CLOSED AND OPEN CURVES
}

\author{
P.A. KRUTITSKII \\ Dept. of Mathematics, Faculty of Physics, Moscow State University, \\ Moscow 119899, Russia. \\ (Received September 23, 1996 and in revised form April 17, 1997)

\begin{abstract}
The Neumann problem for the dissipative Helmholtz equation in a connected plane region bounded by closed and open curves is studied. The existence of classical solution is proved by potential theory. The problem is reduced to the Fredholm equation of the second kind, which is uniquely solvable. Our approach holds for both internal and external domains.
\end{abstract}

KEY WORDS AND PHRASES: Helmholtz equation, Neumann problem, boundary integral equation method.

1991 AMS SUBJECT CLASSIFICATION CODES: 35J05, 35J25, 31A25, 78A45.

\section{INTRODUCTION}

The boundary value problems in domains bounded by closed and open curves were not treated in the theory of 2-D PDEs before. Even in the case of Laplace and Helmholtz equations the problems in domains bounded by closed curves [1-2], [5-8] and problems in the exterior of open arcs [5], [9-11] were treated separately, because different methods were used in their analysis. Previously the Neumann problem in the exterior of an open arc was reduced to the hypersingular integral equation [9-10] or to the infinite algebraic system of equations [11], while the Neumann problem in domains bounded by closed curves was reduced to the Fredholm equation of the second kind [1], [6-8]. The combination of these methods in case of domains bounded by closed and open curves leads to the integral equation, which is algebraic or hypersingular on open curves and it is an equation of the second kind with compact integral operators on the closed curves. The integral equation on the whole boundary is too complicated and the general theory of similar equations are not constructed currently. The approach suggested in the present paper enables to reduce the Neumann problem in domains bounded by closed and open curves to the Fredholm integral equation on the whole boundary with the help of the nonclassical angular potential. Since the boundary integral equation is Fredholm, the solvability theorem follows from the uniqueness theorem, which is ensured for the Neumann problem in the case of the dissipative Helmholtz equation This approach is based on [3-4], where the problems in the exterior of open curves were reduced to the Fredholm integral equations using the angular potential.

\section{FORMULATION OF THE PROBLEM}

By a simple open curve we mean a non-closed smooth arc of finite length without selfintersections [5]

In the plane $x=\left(x_{1}, x_{2}\right) \in R^{2}$ we consider the multiply connected domain bounded by simple 
open curves $\Gamma_{1}^{1}, \ldots, \Gamma_{N_{1}}^{1} \in C^{2, \lambda}, \lambda \in(0,1]$, and simple closed curves $\Gamma_{1}^{2}, \ldots, \Gamma_{N_{2}}^{2} \in C^{2,0}$, so that the curves do not have points in common. We will consider both the case of an external domain and the case of an internal domain, when the curve $\Gamma_{1}^{2}$ encloses all other. We put

$$
\Gamma^{1}=\bigcup_{n=1}^{N_{1}} \Gamma_{n}^{1}, \quad \Gamma^{2}=\bigcup_{n=1}^{N_{2}} \Gamma_{n}^{2}, \quad \Gamma=\Gamma^{1} \cup \Gamma^{2} .
$$

The connected domain bounded by $\Gamma^{2}$ will be called $\mathcal{D}$. We assume that each curve $\Gamma_{n}^{k}$ is parametricized by the arc length $s$ :

$\Gamma_{n}^{k}=\left\{x: x=x(s)=\left(x_{1}(s), x_{2}(s)\right), s \in\left[a_{n}^{k}, b_{n}^{k}\right]\right\}, n=1, \ldots, N_{k}, k=1,2$, so that $a_{1}^{1}<b_{1}^{1}<\ldots<a_{N_{1}}^{1}<b_{N_{1}}^{1}<a_{1}^{2}<b_{1}^{2}<\ldots<a_{N_{2}}^{2}<b_{N_{2}}^{2}$ and the domain $\mathcal{D}$ is to the right when the parameter $s$ increases on $\Gamma_{n}^{2}$. Therefore points $x \in \Gamma$ and values of the parameter $s$ are in one-to-one correspondence except $a_{n}^{2}, b_{n}^{2}$, which correspond to the same point $x$ for $n=1, \ldots, N_{2}$. Below the sets of the intervals on the $O s$ axis

$$
\bigcup_{n=1}^{N_{1}}\left[a_{n}^{1}, b_{n}^{1}\right], \bigcup_{n=1}^{N_{2}}\left[a_{n}^{2}, b_{n}^{2}\right], \bigcup_{k=1}^{2} \bigcup_{n=1}^{N_{k}}\left[a_{n}^{k}, b_{n}^{k}\right]
$$

will be denoted by $\Gamma^{1}, \Gamma^{2}$ and $\Gamma$ also.

We put $C^{0}\left(\Gamma_{n}^{2}\right)=\left\{\mathcal{F}(s): \mathcal{F}(s) \in C^{0}\left[a_{n}^{2}, b_{n}^{2}\right], \mathcal{F}\left(a_{n}^{2}\right)=\mathcal{F}\left(b_{n}^{2}\right)\right\}$ and $C^{0}\left(\Gamma^{2}\right)=\bigcap_{n=1}^{N_{2}} C^{0}\left(\Gamma_{n}^{2}\right)$.

The tangent vector to $\Gamma$ at the point $x(s)$ we denote by $\tau_{x}=(\cos \alpha(s), \quad \sin \alpha(s))$, where $\cos \alpha(s)=x_{1}^{\prime}(s), \sin \alpha(s)=x_{2}^{\prime}(s)$. Let $\mathbf{n}_{x}=(\sin \alpha(s),-\cos \alpha(s))$ be a normal vector to $\Gamma$ at $x(s)$. The direction of $\mathbf{n}_{x}$ is chosen such that it will coincide with the direction of $\tau_{x}$ if $\mathbf{n}_{x}$ is rotated anticlockwise through an angle of $\pi / 2$.

We say, that the function $w(x)$ belongs to the smoothness class $\mathbf{K}$ if

1) $w \in C^{0}\left(\overline{\mathcal{D} \backslash \Gamma^{1}}\right) \cap C^{2}\left(\mathcal{D} \backslash \Gamma^{1}\right)$,

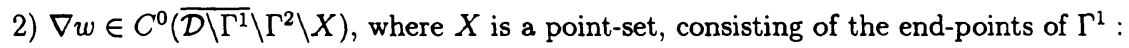

$$
X=\bigcup_{n=1}^{N_{1}}\left(x\left(a_{n}^{1}\right) \cup x\left(b_{n}^{1}\right)\right)
$$

3 ) in the neighborhood of any point $x(d) \in X$ for some constants $\mathcal{C}>0, \epsilon>-1$ the inequality holds

$$
|\nabla w| \leq \mathcal{C}|x-x(d)|^{\epsilon}
$$

where $x \rightarrow x(d)$ and $d=a_{n}^{1}$ or $d=b_{n}^{1}, \quad n=1, \ldots, N_{1}$,

4) there exists a uniform for all $x(s) \in \Gamma^{2}$ limit of $\left(\mathbf{n}_{x}, \nabla_{\bar{x}} w(\bar{x})\right)$ as $\bar{x} \in \mathcal{D} \backslash \Gamma^{1}$ tends to $x \in \Gamma^{2}$ along the normal $\mathbf{n}_{x}$.

REMARK. In the definition of the class $\mathrm{K}$ we consider $\Gamma^{1}$ as a set of cuts in the domain $\mathcal{D}$. According to this definition, $w(x)$ and $\nabla w(x)$ may have a jump across $\Gamma^{1} \backslash X$.

Let us formulate the Neumann problem for the dissipative Helmholtz equation in the domain $\mathcal{D} \backslash \Gamma^{1}$.

PROBLEM U. To find a function $w(x)$ of the class $\mathbf{K}$ which satisfies the Helmholtz equation

$$
w_{x_{1} x_{1}}(x)+w_{x_{2} x_{2}}(x)+\beta^{2} w(x)=0, x \in \mathcal{D} \backslash \Gamma^{1}, \quad \beta=\text { const }, \quad \operatorname{Im} \beta>0
$$

and the boundary condition

$$
\left.\frac{\partial}{\partial \mathbf{n}_{x}} w(x(s))\right|_{\Gamma}=f(s) .
$$


If $\mathcal{D}$ is an external domain, then we add the following condition at infinity

$$
w=o\left(|x|^{-1 / 2}\right), \quad|\nabla w(x)|=o\left(|x|^{-1 / 2}\right), \quad|x|=\sqrt{x_{1}^{2}+x_{2}^{2}} \rightarrow \infty .
$$

All conditions of the problem $U$ must be satisfied in the classical sense. By $\partial w / \partial \mathbf{n}_{x}$ on $\Gamma^{2}$ we mean the limit ensured in the point 4 ) of the definition of the smoothness class $K$. The normal derivative $\partial w / \partial \mathbf{n}_{x}$ has to be continuous across $\Gamma^{1} \backslash X$ and has to take given values on $\Gamma^{1} \backslash X$. At the same time $w(x)$ may have a jump across $\Gamma^{1} \backslash X$.

On the basis of the energy equalities and the technique of equidistant curves [6], we can easily prove the following assertion.

THEOREM 1. If $\Gamma^{1} \in C^{2, \lambda}, \lambda \in(0,1], \Gamma^{2} \in C^{2,0}$, then the problem $\mathrm{U}$ has at most one solution.

The theorem holds for both internal and external domain $\mathcal{D}$.

\section{INTEGRAL EQUATIONS AT THE BOUNDARY}

Below we assume that $f(s)$ from $(2.2 \mathrm{~b})$ is an arbitrary function from the Banach space $C^{0, \lambda}\left(\Gamma^{1}\right) \cap C^{0}\left(\Gamma^{2}\right), \lambda \in(0,1]$.

If $\mathcal{B}_{1}\left(\Gamma^{1}\right), \mathcal{B}_{2}\left(\Gamma^{2}\right)$ are Banach spaces of functions given on $\Gamma^{1}$ and $\Gamma^{2}$, then for functions given on $\Gamma$ we introduce the Banach space $\mathcal{B}_{1}\left(\Gamma^{1}\right) \cap \mathcal{B}_{2}\left(\Gamma^{2}\right)$ with the norm $\|\cdot\|_{\mathcal{B}_{1}\left(\Gamma^{1}\right) \cap B_{2}\left(\Gamma^{2}\right)}=\|\cdot\|_{\mathcal{B}_{1}\left(\Gamma^{1}\right)}+$ $\|\cdot\|_{\mathcal{B}_{2}\left(\Gamma^{2}\right)}$.

By $\int_{\Gamma^{k}} \ldots d \sigma$ we mean $\sum_{n=1}^{N_{k}} \int_{a_{n}^{k}}^{b_{n}^{k}} \ldots d \sigma$.

We consider the angular potential from [3], [4] for the equation $(2.2 a)$ on $\Gamma^{1}$

$$
w_{1}[\mu](x)=\frac{i}{4} \int_{\Gamma^{1}} \mu(\sigma) V(x, \sigma) d \sigma .
$$

The kernel $V(x, \sigma)$ is defined on the each curve $\Gamma_{n}^{1}, n=1, \ldots, N_{1}$ by the formula

$$
V(x, \sigma)=\int_{a_{n}^{1}}^{\sigma} \frac{\partial}{\partial \mathbf{n}_{y}} \mathcal{H}_{0}^{(1)}(\beta|x-y(\xi)|) d \xi, \quad \sigma \in\left[a_{n}^{1}, b_{n}^{1}\right],
$$

where $\mathcal{H}_{0}^{(1)}(z)$ is the Hankel function of the first kind

$$
\begin{aligned}
& \mathcal{H}_{0}^{(1)}(z)=\frac{\sqrt{2} \exp (i z-i \pi / 4)}{\pi \sqrt{z}} \int_{0}^{\infty} \exp (-t) t^{-1 / 2}\left(1+\frac{i t}{2 z}\right)^{-1 / 2} d t \\
& y=y(\xi)=\left(y_{1}(\xi), y_{2}(\xi)\right),|x-y(\xi)|=\sqrt{\left(x_{1-} y_{1}(\xi)\right)^{2}+\left(x_{2}-y_{2}(\xi)\right)^{2}}
\end{aligned}
$$

Below we suppose that $\mu(\sigma)$ belongs to the Banach space $C_{q}^{\omega}\left(\Gamma^{1}\right), \omega \in(0,1], q \in[0,1)$ and satisfies the following additional conditions

$$
\int_{a_{n}^{1}}^{b_{n}^{1}} \mu(\sigma) d \sigma=0, \quad n=1, \ldots, N_{1}
$$

We say, that $\mu(s) \in C_{q}^{\omega}\left(\Gamma^{1}\right)$ if

$$
\mu(s) \prod_{n=1}^{N_{1}}\left|s-a_{n}^{1}\right|^{q}\left|s-b_{n}^{1}\right|^{q} \in C^{0, \omega}\left(\Gamma^{1}\right),
$$


where $C^{0, \omega}\left(\Gamma^{1}\right)$ is a Holder space with the index $\omega$ and

$$
\|\mu(s)\|_{C_{q}^{\omega}\left(\Gamma^{1}\right)}=\left\|\mu(s) \prod_{n=1}^{N_{1}}\left|s-a_{n}^{1}\right|^{q}\left|s-b_{n}^{1}\right|^{q}\right\|_{C^{0, \omega}\left(\Gamma^{1}\right)} .
$$

As shown in [3], [4] for such $\mu(\sigma)$ the angular potential $w_{1}[\mu](x)$ belongs to the class $\mathbf{K}$. In particular, the inequality (2.1) holds with $\epsilon=-q$, if $q \in(0,1)$. Moreover, integrating $w_{1}[\mu](x)$ by parts and using (3.2) we express the angular potential in terms of a double layer potential

$$
w_{1}[\mu](x)=-\frac{i}{4} \int_{\Gamma^{1}} \rho(\sigma) \frac{\partial}{\partial \mathbf{n}_{y}} \mathcal{H}_{0}^{(1)}(\beta|x-y(\sigma)|) d \sigma,
$$

with the density

$$
\rho(\sigma)=\int_{a_{n}^{1}}^{\sigma} \mu(\xi) d \xi, \quad \sigma \in\left[a_{n}^{1}, b_{n}^{1}\right], \quad n=1, \ldots, N_{1} .
$$

Consequently, $w_{1}[\mu](x)$ satisfies both equation $(2.2 \mathrm{a})$ outside $\Gamma^{1}$ and the conditions at infinity (2.2c).

Let us construct a solution of the problem $U$. This solution can be obtained with the help of potential theory for the Helmholtz equation (2.2a). We seek a solution of the problem in the form of the angular potential on $\Gamma^{1}$ and the single-layer potential on $\Gamma^{2}$

$$
w[\mu](x)=w_{1}[\mu](x)+w_{2}[\mu](x),
$$

where $w_{1}[\mu](x)$ is given by $(3.1),(3.3)$ and

$$
w_{2}[\mu](x)=\frac{i}{4} \int_{\Gamma^{2}} \mu(\sigma) \mathcal{H}_{0}^{(1)}(\beta|x-y(\sigma)|) d \sigma .
$$

We will seek $\mu(s)$ from the Banach space $C_{q}^{\omega}\left(\Gamma^{1}\right) \cap C^{0}\left(\Gamma^{2}\right), \omega \in(0,1], q \in[0,1)$ with the norm $\|\cdot\|_{C_{q}^{\omega}\left(\Gamma^{1}\right) \cap C^{0}\left(\Gamma^{2}\right)}=\|\cdot\|_{C_{q}^{\omega}\left(\Gamma^{1}\right)}+\|\cdot\|_{C^{0}\left(\Gamma^{2}\right)}$. Besides, $\mu(s)$ must satisfy conditions (3.2).

It follows from the properties of potentials [1], [3-4], [6], that for such $\mu(s)$ the function (3.5) belongs to the class $\mathbf{K}$ and satisfies all conditions of the problem $\mathbf{U}$ except the boundary condition (2.2b). In the case of the external domain $\mathcal{D}$ the function (3.5) satisfies the condition at infinity (2.2c).

To satisfy the boundary condition we put (3.5) in (2.2b), use the limit formulas for the angular potential from [3] and arrive at the integral equation for the density $\mu(s)$ :

$$
\begin{gathered}
-\frac{1}{2 \pi} \int_{\Gamma^{1}} \mu(\sigma) \frac{\sin \varphi_{0}(x(s), y(\sigma))}{|x(s)-y(\sigma)|} d \sigma+\frac{i}{4} \int_{\Gamma^{1}} \mu(\sigma) \frac{\partial}{\partial \mathbf{n}_{x}} V_{0}(x(s), \sigma) d \sigma-\frac{1}{2} \delta(s) \mu(s)+ \\
+\frac{i}{4} \int_{\Gamma^{2}} \mu(\sigma) \frac{\partial}{\partial \mathbf{n}_{x}} \mathcal{H}_{0}^{(1)}(\beta|x(s)-y(\sigma)|) d \sigma=f(s), \quad s \in \Gamma,
\end{gathered}
$$

where $\delta(s)=0$ if $s \in \Gamma^{1}$ and $\delta(s)=1$ if $s \in \Gamma^{2}$,

$$
\begin{gathered}
V_{0}(x, \sigma)=\int_{a_{n}^{1}}^{\sigma} \frac{\partial}{\partial \mathbf{n}_{y}} h(\beta|x-y(\xi)|) d \xi, \quad \sigma \in\left[a_{n}^{1}, b_{n}^{1}\right], \quad n=1,2, \ldots, N_{1}, \\
h(z)=\mathcal{H}_{0}^{(1)}(z)-\frac{2 i}{\pi} \ln \frac{z}{\beta} .
\end{gathered}
$$

By $\varphi_{0}(x, y)$ we denote the angle between the vector $\overrightarrow{x y}$ and the direction of the normal $\mathbf{n}_{x}$. The angle $\varphi_{0}(x, y)$ is taken to be positive if it is measured anticlockwise from $\mathbf{n}_{x}$ and negative if it is measured clockwise from $\mathbf{n}_{x}$. Besides, $\varphi_{0}(x, y)$ is continuous in $x, y \in \Gamma$ if $x \neq y$. 
Thus, if $\mu(s)$ is a solution of equations (3.2), (3.6) from the space $C_{q}^{\omega}\left(\Gamma^{1}\right) \cap C^{0}\left(\Gamma^{2}\right), \omega \in(0,1], q \in[0,1)$, then the potential (3.5) satisfies all conditions of the problem $\mathrm{U}$. The following theorem holds.

THEOREM 2. If $\Gamma^{1} \in C^{2, \lambda}, \Gamma^{2} \in C^{2,0}, f(s) \in C^{0, \lambda}\left(\Gamma^{1}\right) \cap C^{0}\left(\Gamma^{2}\right), \lambda \in(0,1]$, equation (3.6) has a solution $\mu(s)$ from the Banach space $C_{q}^{\omega}\left(\Gamma^{1}\right) \cap C^{0}\left(\Gamma^{2}\right), \omega \in(0,1], q \in[0,1)$ and condztions (3.2) hold, then the function (3.5) is a solution of the problem $\mathrm{U}$.

Below we look for $\mu(s)$ in the Banach space $C_{q}^{\omega}\left(\Gamma^{1}\right) \cap C^{0}\left(\Gamma^{2}\right)$.

If $s \in \Gamma^{2}$, then (3.6) is an equation of the second kind with compact integral operators. If $s \in \Gamma^{1}$, then (3.6) is a singular integral equation [5].

Our further treatment will be aimed to the proof of the solvability of the system (3.2), (3.6) in the Banach space $C_{q}^{\omega}\left(\Gamma^{1}\right) \cap C^{0}\left(\Gamma^{2}\right)$. Moreover, we reduce the system (3.2), (3.6) to a Fredholm equation of the second kind, which can be easily computed by classical methods.

Equation (3.6) on $\Gamma^{2}$ we rewrite in the form

$$
\mu(s)+\int_{\Gamma} \mu(\sigma) A_{2}(s, \sigma) d \sigma=-2 f(s), s \in \Gamma^{2},
$$

where

$$
A_{2}(s, \sigma)=-\left\{\frac{i}{2}(1-\delta(\sigma)) \frac{\partial}{\partial \mathbf{n}_{x}} V(x(s), \sigma)+\frac{i}{2} \delta(\sigma) \frac{\partial}{\partial \mathbf{n}_{x}} \mathcal{H}_{0}^{(1)}(\beta|x(s)-y(\sigma)|)\right\}
$$

and $V(x, \sigma)$ is the kernel of the angular potential (3.1).

We note $A_{2}(s, \sigma) \in C^{0}\left(\Gamma^{2} \times \Gamma\right)$, because $\Gamma^{2} \in C^{2,0}$.

It can be easily proved that

$$
\frac{\sin \varphi_{0}(x(s), y(\sigma))}{|x(s)-y(\sigma)|}-\frac{1}{\sigma-s} \in C^{0, \lambda}\left(\Gamma^{1} \times \Gamma^{1}\right)
$$

(see [3], [4] for details). Therefore we can rewrite (3.6) on $\Gamma^{1}$ in the form

$$
\frac{1}{\pi} \int_{\Gamma^{1}} \mu(\sigma) \frac{d \sigma}{\sigma-s}+\int_{\Gamma} \mu(\sigma) Y(s, \sigma) d \sigma=-2 f(s), s \in \Gamma^{1},
$$

where

$$
\begin{aligned}
Y(s, \sigma)=\{( & 1-\delta(\sigma))\left[\frac{1}{\pi}\left(\frac{\sin \varphi_{0}(x(s), y(\sigma))}{|x(s)-y(\sigma)|}-\frac{1}{\sigma-s}\right)-\frac{i}{2} \frac{\partial}{\partial \mathbf{n}_{x}} V_{0}(x(s), \sigma)\right]- \\
& \left.-\frac{i}{2} \delta(\sigma) \frac{\partial}{\partial \mathbf{n}_{x}} \mathcal{H}_{0}^{(1)}(\beta|x(s)-y(\sigma)|)\right\} \in C^{0, p_{0}}\left(\Gamma^{1} \times \Gamma\right),
\end{aligned}
$$

$p_{0}=\lambda$ if $0<\lambda<1$ and $p_{0}=1-\epsilon_{0}$ for any $\epsilon_{0} \in(0,1)$ if $\lambda=1$.

\section{THE FREDHOLM INTEGRAL EQUATION}

\section{AND THE SOLUTION OF THE PROBLEM}

Inverting the singular integral operator in (3.8) we arrive at the following integral equation of the second kind [5]:

$$
\mu(s)+\frac{1}{Q_{1}(s)} \int_{\Gamma} \mu(\sigma) A_{0}(s, \sigma) d \sigma+\frac{1}{Q_{1}(s)} \sum_{n=0}^{N_{1}-1} G_{n} s^{n}=\frac{1}{Q_{1}(s)} \Phi_{0}(s), \quad s \in \Gamma^{1},
$$

where $G_{0}, \ldots, G_{N_{1}-1}$ are arbitrary constants and

$$
A_{0}(s, \sigma)=-\frac{1}{\pi} \int_{\Gamma^{1}} \frac{Y(\xi, \sigma)}{\xi-s} Q_{1}(\xi) d \xi, \quad \Phi_{0}(s)=\frac{1}{\pi} \int_{\Gamma^{1}} \frac{2 Q_{1}(\sigma) f(\sigma)}{\sigma-s} d \sigma
$$




$$
Q_{1}(s)=\prod_{n=1}^{N_{1}}\left|\sqrt{s-a_{n}^{1}} \sqrt{b_{n}^{1}-s}\right| \operatorname{sign}\left(s-a_{n}^{1}\right) .
$$

To derive equations for $G_{0}, \ldots, G_{N_{1}-1}$ we substitute $\mu(s)$ from (4.1) in the conditions (3.2), then we obtain

$$
\int_{\Gamma} \mu(\sigma) l_{n}(\sigma) d \sigma+\sum_{m=0}^{N_{1}-1} B_{n m} G_{m}=H_{n}, \quad n=1, \ldots, N_{1},
$$

where

$$
\begin{gathered}
l_{n}(\sigma)=-\int_{\Gamma_{n}^{1}} Q_{1}^{-1}(s) A_{0}(s, \sigma) d s, \quad H_{n}=-\int_{\Gamma_{n}^{1}} Q_{1}^{-1}(s) \Phi_{0}(s) d s \\
B_{n m}=-\int_{\Gamma_{n}^{1}} Q_{1}^{-1}(s) s^{m} d s
\end{gathered}
$$

By $B$ we denote the $N_{1} \times N_{1}$ matrix with the elements $B_{n m}$ from (4.3). As shown in [4], the matrix $B$ is invertible. The elements of the inverse matrix will be called $\left(B^{-1}\right)_{n m}$. Inverting the matrix $B$ in (4.2) we express the constants $G_{0}, \ldots, G_{N_{1}-1}$ in terms of $\mu(s)$

$$
G_{n}=\sum_{m=1}^{N_{1}}\left(B^{-1}\right)_{n m}\left[H_{m}-\int_{\Gamma} \mu(\sigma) l_{m}(\sigma) d \sigma\right] .
$$

We substitute $G_{n}$ in (4.1) and obtain the integral equation for $\mu(s)$ on $\Gamma^{1}$

$$
\mu(s)+\frac{1}{Q_{1}(s)} \int_{\Gamma} \mu(\sigma) A_{1}(s, \sigma) d \sigma=\frac{1}{Q_{1}(s)} \Phi_{1}(s), s \in \Gamma^{1},
$$

where

$$
\begin{gathered}
A_{1}(s, \sigma)=A_{0}(s, \sigma)-\sum_{n=0}^{N_{1}-1} s^{n} \sum_{m=1}^{N_{1}}\left(B^{-1}\right)_{n m} l_{m}(\sigma), \\
\Phi_{1}(s)=\Phi_{0}(s)-\sum_{n=0}^{N_{1}-1} s^{n} \sum_{m=1}^{N_{1}}\left(B^{-1}\right)_{n m} H_{m} .
\end{gathered}
$$

It can be shown using the properties of singular integrals [2], [5], that $\Phi_{0}(s), A_{0}(s, \sigma)$ are Holder functions if $s \in \Gamma^{1}, \sigma \in \Gamma$. Therefore, $\Phi_{1}(s), A_{1}(s, \sigma)$ are also Holder functions if $s \in \Gamma^{1}, \sigma \in \Gamma$. Consequently, any solution of (4.4) belongs to $C_{1 / 2}^{\omega}\left(\Gamma^{1}\right)$ and below we look for $\mu(s)$ on $\Gamma^{1}$ in this space.

We put $Q(s)=(1-\delta(s)) Q_{1}(s)+\delta(s), s \in \Gamma$.

Instead of $\mu(s) \in C_{1 / 2}^{\omega}\left(\Gamma^{1}\right) \cap C^{0}\left(\Gamma^{2}\right)$ we introduce the new unknown function $\mu_{*}(s)=\mu(s) Q(s) \in$ $C^{0, \omega}\left(\Gamma^{1}\right) \cap C^{0}\left(\Gamma^{2}\right)$ and rewrite (3.7), (4.4) in the form of one equation

$$
\mu_{*}(s)+\int_{\Gamma} \mu_{*}(\sigma) Q^{-1}(\sigma) A(s, \sigma) d \sigma=\Phi(s), \quad s \in \Gamma,
$$

where

$$
A(s, \sigma)=(1-\delta(s)) A_{1}(s, \sigma)+\delta(s) A_{2}(s, \sigma), \quad \Phi(s)=(1-\delta(s)) \Phi_{1}(s)-2 \delta(s) f(s) .
$$

Thus, the system of equations (3.2), (3.6) for $\mu(s)$ has been reduced to the equation (45) for the function $\mu_{*}(s)$. It is clear from our consideration that any solution of (4.5) gives a solution of system (3.2), (3.6).

As noted above, $\Phi_{1}(s)$ and $A_{1}(s, \sigma)$ are Holder functions if $s \in \Gamma^{1}, \sigma \in \Gamma$. More precisely (see [4], [5]), $\Phi_{1}(s) \in C^{0, p}\left(\Gamma^{1}\right), \quad p=\min \{1 / 2, \lambda\}$ and $A_{1}(s, \sigma)$ belongs to $C^{0, p}\left(\Gamma^{1}\right)$ in $s$ uniformly with respect to $\sigma \in \Gamma$. We arrive at the following assertion. 
LEMMA. If $\Gamma^{1} \in C^{2, \lambda}, \lambda \in(0,1], \Gamma^{2} \in C^{2,0}, \Phi(s) \in C^{0, p}\left(\Gamma^{1}\right) \cap C^{0}\left(\Gamma^{2}\right), \quad p=\min \{\lambda, 1 / 2\}$, and $\mu_{*}(s)$ from $C^{0}(\Gamma)$ satisfies the equation $(4.5)$, then $\mu_{*}(s) \in C^{0, p}\left(\Gamma^{1}\right) \cap C^{0}\left(\Gamma^{2}\right)$.

The condition $\Phi(s) \in C^{0, p}\left(\Gamma^{1}\right) \cap C^{0}\left(\Gamma^{2}\right)$ holds if $f(s) \in C^{0, \lambda}\left(\Gamma^{1}\right) \cap C^{0}\left(\Gamma^{2}\right)$.

Hence below we will seek $\mu_{*}(s)$ from $C^{0}(\Gamma)$.

Since $A(s, \sigma) \in C^{0}(\Gamma \times \Gamma)$, the integral operator from (4.5):

$$
\mathbf{A} \mu_{*}=\int_{\Gamma} \mu_{*}(\sigma) Q^{-1}(\sigma) A(s, \sigma) d \sigma
$$

is a compact operator mapping $C^{0}(\Gamma)$ into itself. Therefore, (4.5) is a Fredholm equation of the second kind in the Banach space $C^{0}(\Gamma)$.

Let us show that homogeneous equation (4.5) has only a trivial solution. Then, according to Fredholm's theorems, the inhomogeneous equation (4.5) has a unique solution for any right-hand side. We will prove this by a contradiction. Let $\mu_{*}^{0}(s) \in C^{0}(\Gamma)$ be a non-trivial solution of the homogeneous equation (4.5). According to the lemma $\mu_{*}^{0}(s) \in C^{0, p}\left(\Gamma^{1}\right) \cap C^{0}\left(\Gamma^{2}\right), \quad p=\min \{\lambda, 1 / 2\}$. Therefore the function $\mu^{0}(s)=\mu_{*}^{0}(s) Q^{-1}(s) \in C_{1 / 2}^{p}\left(\Gamma^{1}\right) \cap C^{0}\left(\Gamma^{2}\right)$ converts the homogeneous equations (3.7), (4.4) into identities. Using the homogeneous identity (4.4) we check, that $\mu^{0}(s)$ satisfies conditions (3.2). Besides, acting on the homogeneous identity (4.4) with a singular operator with the kernel $(s-t)^{-1}$ we find that $\mu^{0}(s)$ satisfies the homogeneous equation (3.8). Consequently, $\mu^{0}(s)$ satisfies the homogeneous equation (3.6). On the basis of theorem $2, w\left[\mu^{0}\right](x)$ is a solution of the homogeneous problem $\mathrm{U}$. According to theorem 1: $w\left[\mu^{0}\right](x) \equiv 0, x \in \mathcal{D} \backslash \Gamma^{1}$. Using the limit formulas for tangent derivatives of an angular potential [3], we obtain

$$
\lim _{x \rightarrow x(s) \in\left(\Gamma^{1}\right)^{+}} \frac{\partial}{\partial \tau_{x}} w\left[\mu^{0}\right](x)-\lim _{x \rightarrow x(s) \in\left(\Gamma^{1}\right)^{-}} \frac{\partial}{\partial \tau_{x}} w\left[\mu^{0}\right](x)=\mu^{0}(s) \equiv 0, s \in \Gamma^{1} .
$$

By $\left(\Gamma^{1}\right)^{+}$we denote the side of $\Gamma^{1}$ which is on the left as a parameter $s$ increases and by $\left(\Gamma^{1}\right)^{-}$we denote the other side.

Hence, $w\left[\mu^{0}\right](x)=w_{2}\left[\mu^{0}\right](x) \equiv 0, \quad x \in \mathcal{D}$, and $\mu^{0}(s)$ satisfies the following homogeneous equation

$$
-\frac{1}{2} \mu^{0}(s)+\frac{i}{4} \int_{\Gamma^{2}} \mu^{0}(\sigma) \frac{\partial}{\partial \mathbf{n}_{x}} \mathcal{H}_{0}^{(1)}(\beta|x(s)-y(\sigma)|) d \sigma=0, s \in \Gamma^{2} .
$$

The Fredholm equation (4.6) is well-known in classical mathematical physics [1], [6]. We arrive at (4.6) when solving the Neumann problem for the Helmholtz equation (2.2a) in the domain $\mathcal{D}$ by the single layer potential. It is well-known [1], that the equation (4.6) has only the trivial solution $\mu^{0}(s) \equiv 0$ in $C^{0}\left(\Gamma^{2}\right)$. This is true for both internal and external domain $\mathcal{D}$.

Consequently, if $s \in \Gamma$, then $\mu^{0}(s) \equiv 0, \mu_{*}^{0}(s)=\mu^{0}(s) Q^{-1}(s) \equiv 0$ and we arrive at the contradiction to the assumption that $\mu_{*}^{0}(s)$ is a non-trivial solution of the homogeneous equation (4.5). Thus, the homogeneous Fredholm equation (4.5) has only a trivial solution in $C^{0}(\Gamma)$. We have proved the following theorem.

THEOREM 3. If $\Gamma^{1} \in C^{2, \lambda}, \Gamma^{2} \in C^{2,0}, \lambda \in(0,1]$, then (4.5) is a Fredholm equation of the second kind in the space $C^{0}(\Gamma)$. Moreover, equation (4.5) has a unique solution $\mu_{*}(s) \in C^{0}(\Gamma)$ for any $\Phi(s) \in C^{0}(\Gamma)$.

As a consequence of the theorem 3 and the lemma we obtain the corollary.

COROLLARY. If $\Gamma^{1} \in C^{2, \lambda}, \lambda \in(0,1], \Gamma^{2} \in C^{2,0}$ and $\Phi(s) \in C^{0, p}\left(\Gamma^{1}\right) \cap C^{0}\left(\Gamma^{2}\right)$, where $p=\min \{\lambda, 1 / 2\}$, then the unique solution of (4.5) in $C^{0}(\Gamma)$, ensured by theorem 3 , belongs to $C^{0, p}\left(\Gamma^{1}\right) \cap C^{0}\left(\Gamma^{2}\right)$.

We recall that $\Phi(s)$ belongs to the class of smoothness required in the corollary if $f(s) \in$ $C^{0, \lambda}\left(\Gamma^{1}\right) \cap C^{0}\left(\Gamma^{2}\right)$. As mentioned above, if $\mu_{*}(s) \in C^{0, p}\left(\Gamma^{1}\right) \cap C^{0}\left(\Gamma^{2}\right)$ is a solution of (4.5), then 
$\mu(s)=\mu_{*}(s) Q^{-1}(s) \in C_{1 / 2}^{p}\left(\Gamma^{1}\right) \cap C^{0}\left(\Gamma^{2}\right)$ is a solution of system (3.2), (3.6). We obtain the following statement.

THEOREM 4. If $\Gamma^{1} \in C^{2, \lambda}, \Gamma^{2} \in C^{2,0}, f(s) \in C^{0, \lambda}\left(\Gamma^{1}\right) \cap C^{0}\left(\Gamma^{2}\right), \lambda \in(0,1]$, then the system of equations (3.2), (3.6) has a solution $\mu(s) \in C_{1 / 2}^{p}\left(\Gamma^{1}\right) \cap C^{0}\left(\Gamma^{2}\right), p=\min \{1 / 2, \lambda\}$, which is expressed by the formula $\mu(s)=\mu_{*}(s) Q^{-1}(s)$, where $\mu_{*}(s) \in C^{0, p}\left(\Gamma^{1}\right) \cap C^{0}\left(\Gamma^{2}\right)$ is the unique solution of the Fredholm equation (4.5) in $C^{0}(\Gamma)$.

On the basis of the theorem 2 we arrive at the final result.

THEOREM 5. If $\Gamma^{1} \in C^{2, \lambda}, \Gamma^{2} \in C^{2,0}, f(s) \in C^{0, \lambda}\left(\Gamma^{1}\right) \cap C^{0}\left(\Gamma^{2}\right), \lambda \in(0,1]$, then the solution of the problem $\mathrm{U}$ exists and is given by (3.5), where $\mu(s)$ is a solution of equations (3.2), (3.6) from $C_{1 / 2}^{p}\left(\Gamma^{1}\right) \cap C^{0}\left(\Gamma^{2}\right), p=\min \{1 / 2, \lambda\}$ ensured by the theorem 4.

It can be checked directly that the solution of the problem $U$ satisfies condition (2.1) with $\epsilon=-1 / 2$. Explicit expressions for singularities of the solution gradient at the end-points of the open curves can be easily obtained with the help of formulas presented in [4].

\section{REFERENCES}

[1] COLTON, D. and KRESS R., Integral equation methods in scattering theory, John Wiley \& Sons, N.Y., 1983.

[2] GAKHOV, F.D., Boundary value problems, Pergamon Press, Oxford; Addison-Wesley, Reading, Mass., 1966.

[3] KRUTITSKII, P.A., Dirichlet problem for the Helmholtz equation outside cuts in a plane, Comp. Maths. Math. Phys. 34 (1994), 1073-1090.

[4] KRUTITSKII, P.A., Neumann problem for the Helmholtz equation outside cuts in a plane, Comp. Maths. Math. Phys. 34 (1994), 1421-1431.

[5] MUSKHELISHVILI, N.I., Singular integral equations, Noordhoff, Groningen, 1972.

[6] VLADIMIROV, V.S., Equations of mathematical physics, Marcel Dekker, N.Y., 1971.

[7] TORRES, R.H. and WELLAND, G.V., The Helmholtz equation and transmission problems with Lipschitz interfaces, Indiana Univ. Math. J. 42 (1993), 1457-1485.

[8] PETERSDORF, T.V., Boundary integral equations for mixed Dirichlet, Neumann and transmission problems, Math. Meth. Appl. Sci. 11 (1989), 185-213.

[9] DURAND, M., Layer potentials and boundary value problems for the Helmholtz equation in the complement of a thin obstacle, Math. Meth. Appl. Sci. 5 (1983), 389-421.

[10] PANASYUK, V.V., SAVRUK, M.P. and NAZARCHUK, Z.T., The method of singular entegral equations in two-dimensional diffraction problems, Naukova Dumka, Kiev, 1984. (in Russian).

[11] TUCHKIN, Y. A., Scattering of waves by an unclosed cylindrical screen of an arbitrary profile with Neumann boundary condition. Dokl. Akad. Nauk SSSR 293 (1987), 343-345. (in Russian). 


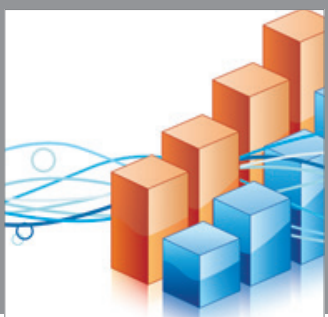

Advances in

Operations Research



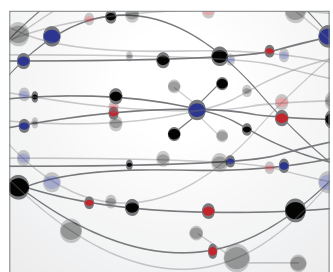

\section{The Scientific} World Journal
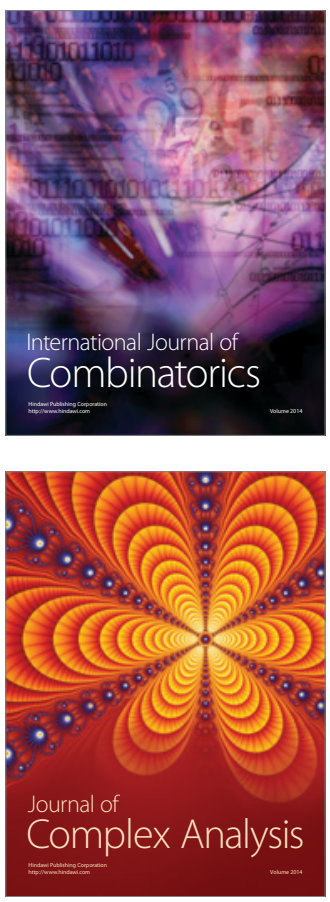

International Journal of

Mathematics and

Mathematical

Sciences
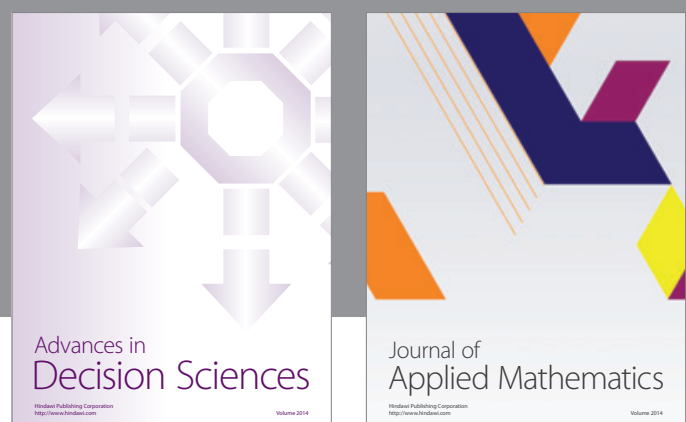

Journal of

Applied Mathematics
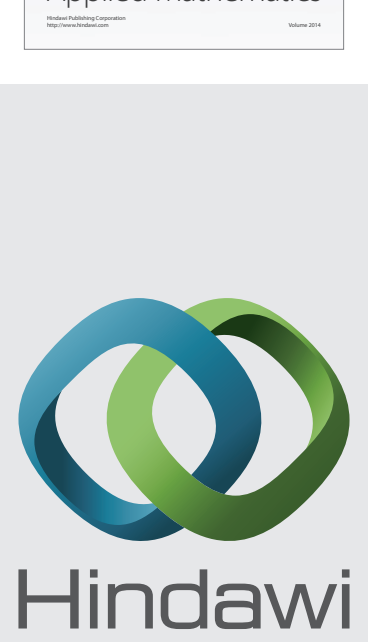

Submit your manuscripts at http://www.hindawi.com


Mathematical Problems in Engineering
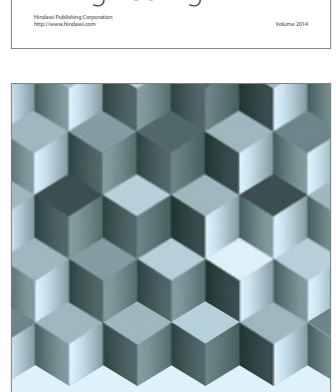

Journal of

Function Spaces


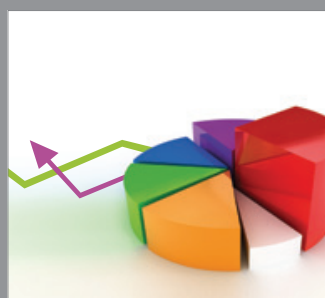

ournal of

Probability and Statistics

Promensencen
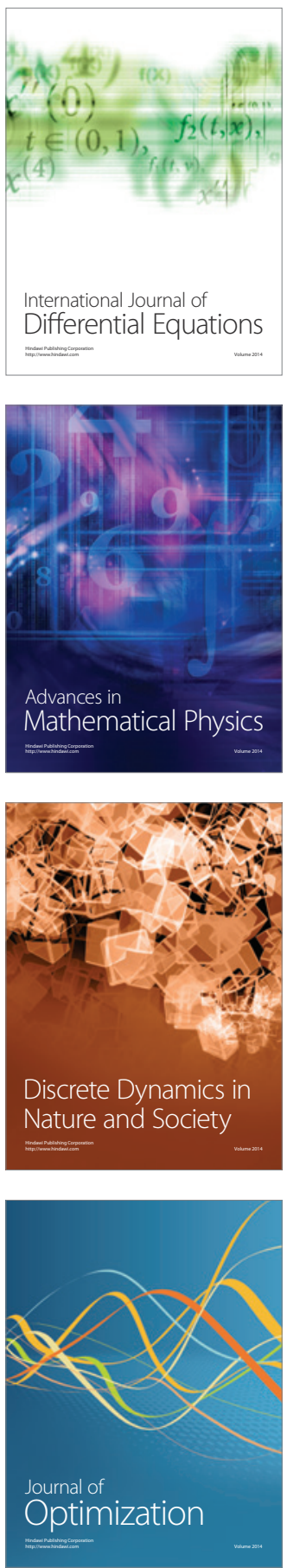